\title{
Nuclear Analysis for Near Term Fusion Devices
}

\section{Final Technical Report}

M. Sawan, G. Kulcinski, and D. Henderson

Fusion Technology Institute

University of Wisconsin - Madison

April 2, 2007 


\section{Introduction}

A Next Step Options (NSO) study was initiated to consider the logical steps that might be undertaken to restructure the U.S. Fusion Sciences Program. Most of the effort was concentrated on designing the Fusion Ignition Research Experiment (FIRE), which is in the preconceptual design phase. It utilizes 16 cryogenically cooled wedged copper TF coils with beryllium copper in the inner legs and OFHC copper in the outer legs. The UW-FTI staff provided significant contributions in the areas of neutronics, shielding and activation analyses. The design went through different changes. Early in the year 2002 the baseline design changed from a major radius of $2 \mathrm{~m}$ to a major radius of $2.14 \mathrm{~m}$ and an aspect ratio of 3.6. In addition the fusion power during the DT pulses changed from $200 \mathrm{MW}$ to $150 \mathrm{MW}$. We spent significant part of the effort calculating the nuclear performance parameters for the final baseline design. While pulses producing a total of $5 \mathrm{TJ}$ of DT fusion energy and $0.5 \mathrm{TJ}$ of DD fusion energy were considered in the previous designs, a detailed experimental plan was developed that results in higher total fusion energy. We assessed the impact on the peak magnet insulator dose. Multi-dimensional calculations were performed also to determine the impact of plasma shape and profile on he peak radiation effects in the TF coils. We performed multidimensional calculations for one of the most critical diagnostics ports to assess streaming and determine the nuclear environment at the sensitive components. The radwaste level and volume was quantified for the different components of FIRE. We updated the neutronics and activation sections of the FIRE Engineering Report to include the latest results. A summary of the activities performed along with the significant findings is given in this report.

\section{Nuclear Parameters for the FIRE Baseline Design}

\section{II.1 Nuclear Heating}

Nuclear heating deposited in the different components was determined for the DT pulses with the largest fusion power of $150 \mathrm{MW}$. Table 1 gives the peak power density values in the different components at the chamber midplane. Nuclear heating was calculated also for the components in the $1.3 \mathrm{~cm}$ diagnostics space behind the tiles. The nuclear heating values in these components at midplane are $25.5 \mathrm{~W} / \mathrm{cm}^{3}$ in $\mathrm{MgO}, 23.7$ $\mathrm{W} / \mathrm{cm}^{3}$ in $\mathrm{SS}$ and $24.9 \mathrm{~W} / \mathrm{cm}^{3}$ in $\mathrm{Cu}$. Calculations were also performed for the outer divertor, which is exposed to the most severe conditions in the divertor region. The results revealed that relatively high nuclear heating of $30 \mathrm{~W} / \mathrm{cm}^{3}$ is deposited in the $\mathrm{W}$ rods with a peak value of only $11 \mathrm{~W} / \mathrm{cm}^{3}$ in the Cu heat sink. The amount of nuclear heating in the TF magnets strongly influences the achievable pulse length. The total nuclear heating in the $16 \mathrm{TF}$ coils during the DT shots is $16 \mathrm{MW}$ with $90 \%$ of it deposited in the lightly shielded IB legs. For the DD pulses with the largest fusion power (1 MW), nuclear heating values are at least two orders of magnitude lower.

Table 1. Peak nuclear heating $\left(\mathrm{W} / \mathrm{cm}^{3}\right)$ at midplane.

\begin{tabular}{|l|c|c|}
\hline & IB & OB \\
\hline Be PFC & 20.65 & 22.07 \\
\hline Cu Tiles & 29.08 & 28.71 \\
\hline Gasket & 25.17 & 25.17 \\
\hline Cooled Cu Vessel Cladding & 24.92 & 24.86 \\
\hline H2O FWCoolant & 17.11 & 19.16 \\
\hline SS Inner VV Wall & 20.96 & 19.16 \\
\hline SS VV Filer & 20.40 & 17.67 \\
\hline H2O VV Coolant & 9.24 & 9.61 \\
\hline SS Outer VV Wall & 18.79 & 0.043 \\
\hline Cu Magnet & 12.09 & 0.012 \\
\hline
\end{tabular}




\section{II.2 Vacuum Vessel Radiation Damage}

Since the vacuum vessel (VV) is protected from the fusion neutrons by the thin FW/tiles, the issue of reweldability was addressed. The end-of-life helium production in the VV structure should be limited to 1 appm to allow for rewelding. For the FIRE goal of cumulative 5 TJ DT and 0.5 TJ DD fusion energy, the peak VV He production values in the IB, OB, and divertor regions are $0.091,0.125$, and 0.013 appm, respectively. The contribution from DD shots is very small $(<0.15 \%)$. The results imply that reweldability of the VV should not be a concern.

\section{II.3 Copper Radiation Damage}

Table 2 gives the end-of-life peak dpa values in the $\mathrm{Cu}$ tiles, VV cladding, $\mathrm{Cu}$ heat sink in outer divertor, and $\mathrm{Cu}$ TF coils for the FIRE baseline design. Although the damage levels are very low, significant effects on physical and mechanical properties might occur. These effects are strongly dependent on irradiation temperature and have been the subject of numerous studies as part of the ITER R\&D program.

Table 2. Peak End-of-Life Cu dpa in FIRE.

\begin{tabular}{|l|c|}
\hline & $\begin{array}{c}\text { Total dpa in } \mathrm{Cu} \text { at } \\
\text { end-of-life }\end{array}$ \\
\hline IB Tiles & 0.0271 \\
\hline OB Tiles & 0.0298 \\
\hline Divertor & 0.0125 \\
\hline IB VV Cladding & 0.0178 \\
\hline OB VV Cladding & 0.0204 \\
\hline Magnet at IB & 0.0055 \\
\hline Magnet at OB & $6.26 \times 10^{-6}$ \\
\hline Magnet at Divertor & $3.78 \times 10^{-4}$ \\
\hline
\end{tabular}

Based on the irradiation levels and operation conditions in FIRE and the available data on Cu alloys, we can identify the R\&D needs as follows:

- Data on loss of ductility of $\mathrm{BeCu}$ (or OFHC) at temperatures between 80 and $373^{\circ} \mathrm{K}$ with doses $<$ 0.01 dpa.

- Data on fatigue, fracture toughness and fatigue crack growth rate behavior in high-strength, highconductivity copper alloys.

- Thermal creep data for $\mathrm{CuCrZr}$ at temperatures up to $500^{\circ} \mathrm{C}$. There is no need to perform irradiation creep measurements on $\mathrm{Cu}$ alloys for the low doses proposed in FIRE.

\section{II.4 Radiation Induced Resistivity in the Copper Conductors of the TF Coils}

The $17510 \mathrm{BeCu}$ alloy is used in the inner legs of the TF coils with 10200 OFHC copper being utilized in the rest of the TF coils. A concern with $\mathrm{Cu}$ magnet conductors is the increased electrical resistivity that impacts the performance of the TF coils through increasing the $\mathrm{I}^{2} \mathrm{R}$ heating and the redistribution of the current across the coil. The temperature at the end of the pulse will increase with possible impact on the achievable pulse length. The increase in electrical resistivity results from solute transmutation products and displacement damage. In a low-fluence machine such as FIRE, resistivity increase is dominated by point defects and defect clusters produced by displacement damage.

Figure 1 shows the resistivity increases at end-of-life from solute transmutation products and displacement damage in the $\mathrm{BeCu}$ alloy as a function of depth in the inner leg of the TF magnet at midplane. It is clear 
that the total resistivity increase is dominated by displacement damage with the resistivity increase from solute transmutation products contributing less than $0.05 \%$ of the total resistivity increase. The resistivity increase drops by a factor of $\sim 30$ across the magnet thickness. Resistivity increase from solute transmutation products contributes less than $0.3 \%$ of the total resistivity increase of the OFHC copper conductor. The spatial distribution of the resistivity results in redistributing the current across the coil.

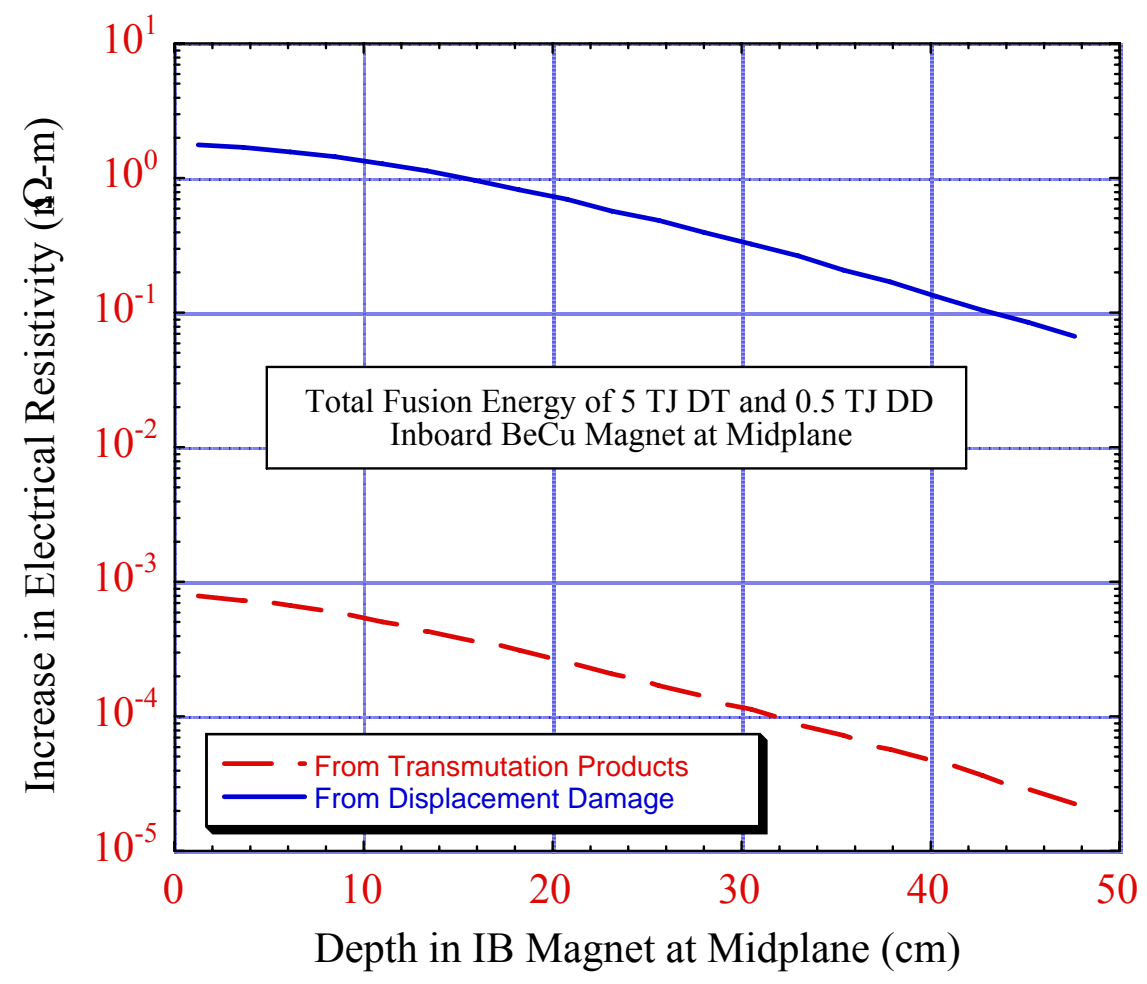

Fig. 1. Resistivity change in $\mathrm{BeCu}$ as a function of depth in magnet at inboard midplane.

The unirradiated resistivity of the $68 \%$ IACS BeCu used in FIRE varies from $\sim 10 \mathrm{n} \Omega$-m at $80 \mathrm{~K}$ to $\sim 30 \mathrm{n} \Omega-$ $\mathrm{m}$ at room temperature. This implies that the maximum increase in resistivity of the $\mathrm{BeCu}$ at end-of-life varies from $\sim 18 \%$ at the start of the pulse to $\sim 6 \%$ at the end of the pulse. The unirradiated resistivity of the 10200 OFHC copper used in FIRE ranges from $\sim 2 \mathrm{n} \Omega-\mathrm{m}$ at $80 \mathrm{~K}$ to $\sim 16 \mathrm{n} \Omega-\mathrm{m}$ at room temperature. Hence, the maximum increase in resistivity of the OFHC copper at end-of-life varies from $\sim 2 \%$ at the start of the pulse to $\sim 0.25 \%$ at the end of the pulse.

\section{II.5 Magnet Insulator Dose}

The insulator dose rate in the TF magnet was calculated at the front layer of the magnet winding pack. For 5 $\mathrm{TJ}$ of DT fusion energy and $0.5 \mathrm{TJ}$ of DD fusion energy, Table 3 provides the peak cumulative magnet insulator dose in the IB, OB, and divertor regions. The peak cumulative magnet insulator dose is $1.05 \times 10^{10}$ Rads in the lightly shielded IB leg at midplane. At this location the fast neutron fluence $(\mathrm{E}>0.1 \mathrm{MeV})$ is $8.1 \times 10^{18} \mathrm{n} / \mathrm{cm}^{2}$ and the total neutron fluence is $1.5 \times 10^{19} \mathrm{n} / \mathrm{cm}^{2}$. About $55 \%$ of the neutron fluence is above $0.1 \mathrm{MeV}$ at the front of the magnet and drops to $35 \%$ at the back of the magnet. The DD shots contribute $13 \%$ of this value. The dose rate decreases by three orders of magnitude as one moves poloidally to the OB midplane. The gamma contribution to the total dose is $35-50 \%$. The dose decreases by an order of magnitude in $\sim 22 \mathrm{~cm}$ of the IB magnet. 
Table 3. Cumulative Peak Magnet Insulator Dose (Rads)

\begin{tabular}{|l|c|c|}
\hline & $\begin{array}{c}\text { Total Dose } \\
\text { (Rads) }\end{array}$ & $\begin{array}{c}\% \text { from DD } \\
\text { Shots }\end{array}$ \\
\hline IB midplane & $1.05 \times 10^{10}$ & $13 \%$ \\
\hline OB midplane & $1.05 \times 10^{7}$ & $1.6 \%$ \\
\hline Divertor & $8.10 \times 10^{8}$ & $10 \%$ \\
\hline
\end{tabular}

The mechanical strength, dielectric strength, and electric resistivity are the important properties that could be affected by irradiation. The shear strength is the property most sensitive to irradiation. The commonly accepted dose limit for epoxies is $10^{9}$ Rads. Polyimides and bismaleimides are more radiation resistant with experimental data showing only a small degradation in shear strength at dose levels in excess of $10^{10}$ Rads. However, they are difficult and expensive to process due to their high viscosity and requirement for high temperatures to fully cure. In addition, they have initial mechanical properties lower than those achievable with epoxies. Hybrids of epoxies, polyimides, bismaleimides, and cyanate esters are being developed to both improve the insulation system's ability to withstand high levels of radiation and to improve the resin system's overall processability, which includes irradiation measurements at dose levels close to those expected in FIRE. The information provided here gives guidance to the insulator development R\&D program regarding the dose levels and the proper mix between neutron and gamma irradiation to insure relevance to the FIRE conditions.

In the FIRE design with wedged coils and added compression ring, the TF inner leg insulation does not have to have significant bond shear strength that is most sensitive to radiation. The peak torsional shear stresses occur at the top and bottom of the IB leg behind the divertor. The end-of-life insulator dose at these locations is reduced to $\sim 10^{9}$ Rads due to the additional shielding provided by the divertor. Based on that, it is expected that insulation materials will be identified that can last for the whole device lifetime with the proposed operation scenario and load conditions.

\section{II.6 Activity and Decay Heat}

Figure 2 shows the specific decay heat values generated in the IB region. The PFC on the FW and divertor produce the highest levels of specific activity and decay heat. However, the operational schedule with several hours between pulses allows for the decay of short-lived radionuclides between pulses, resulting in low levels of activity and decay heat at shutdown. Immediately following DT shots, the largest specific activity is $\sim 62 \mathrm{Ci} / \mathrm{cm}^{3}$ and the largest specific decay heat is $\sim 0.12 \mathrm{~W} / \mathrm{cm}^{3}$. The activity and decay heat generated following DD shots are at least three orders of magnitude lower. The decay heat induced in the FW/tiles, divertor, and $\mathrm{Cu}$ magnet at shutdown is dominated by the copper isotopes ${ }^{62} \mathrm{Cu}\left(\mathrm{T}_{1 / 2}=9.74 \mathrm{~min}\right)$ and ${ }^{66} \mathrm{Cu}\left(\mathrm{T}_{1 / 2}=5.1 \mathrm{~min}\right)$. The decay heat induced in the $\mathrm{VV}$ at shutdown is dominated by the ${ }^{52} \mathrm{~V}\left(\mathrm{~T}_{1 / 2}=3.76 \mathrm{~min}\right)$ and ${ }^{56} \mathrm{Mn}\left(\mathrm{T}_{1 / 2}=2.578 \mathrm{hr}\right)$ isotopes. In general, the short-term activity and decay heat values at shutdown are almost fully dominated by activation during the last pulse.

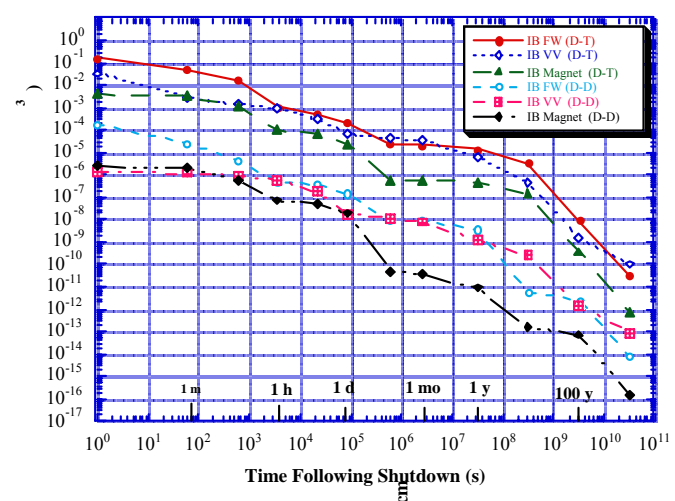

Fig. 2. Decay heat induced in the inboard side. 


\section{Source Geometrical Effects on Peak Nuclear Parameters in FIRE TF Coils}

Neutronics calculations performed using a one-dimensional cylindrical model that includes both the IB and $\mathrm{OB}$ regions account for the toroidal effect and the impact of each region on the nuclear parameters in the other. However, important three-dimensional effects are not included. In the 1-D model the volumetric neutron source in the plasma zone is assumed to be uniform and extended infinitely in the vertical direction. In the real 3-D geometry, the neutron source has finite vertical extension with a spatial profile that peaks near the plasma major radius at the reactor midplane. As a result, the angular distribution of source neutrons incident on the first wall will be quite different. In the 1-D model most source neutrons are incident at a glancing angle compared to the mostly perpendicular distribution in the exact 3-D geometry. Consequently, the 1-D model is expected to yield higher nuclear parameters near the first wall. We performed 2-D calculations to assess the impact of the source geometrical effects on the peak nuclear parameters in the FIRE TF coils occurring at the IB midplane. The correct plasma boundary and neutron source density profile were used is the calculations.

The peak magnet nuclear heating is $9.25 \mathrm{~W} / \mathrm{cm}^{3}$ which is $\sim 23 \%$ lower than that obtained from the $1-\mathrm{D}$ calculations. The total end-of-life cumulative insulator dose was calculated for a total of $5 \mathrm{TJ}$ DT and $0.5 \mathrm{TJ}$ DD fusion energies. The peak insulator dose is $8.71 \times 10^{9}$ Rads which is $\sim 17 \%$ lower than that predicted by the 1-D model. This value drops by a factor of $\sim 4$ as one moves to the top and bottom of the IB leg of the TF coil.

\section{Impact of FIRE Experimental Plan on Peak Magnet Insulator Dose}

The peak insulator dose of $8.71 \times 10^{9}$ Rads is based on a total of 5 TJ DT and 0.5 TJ DD fusion energies. We have $1.57 \times 10^{9}$ Rads per TJ of DT fusion energy and $1.7 \times 10^{9}$ Rads per TJ of DD fusion energy. These values can be used to determine the peak insulator dose for any combination of DT and DD pulses. For the current baseline experimental plan with 6.336 TJ DT and 0.049 TJ DD the peak insulator dose is $10^{10}$ Rads. For the proposed extended operation schedule with 12.771 TJ DT and 0.185 TJ DD the peak dose will be $2.04 \times 10^{10}$ Rads.

\section{Radiation Environment at FIRE Midplane Diagnostic Ports}

Neutron and gamma fluxes can affect plasma diagnostic performance through enhanced conductivity of electrical insulation and scintillation and absorption in optical components close to the tokamak. Determination of the radiation environment is essential for estimating shielding requirements for diagnostic components such as insulated cables, windows, fiberoptics and transducers, as well as detectors and their associated electronics. In addition, streaming through diagnostics penetrations could lead to excessive doses outside the machine.

In FIRE, a few diagnostics, such as the neutral particle analyzer (NPA) and impurity pellet guide tubes require straight holes through the port shielding plugs. Other diagnostics, notably optical systems such as Thomson scattering, will make use of labyrinthine penetrations to curtail the streaming. Such penetrations will include four bends with mirrors at the corners. The midplane diagnostics port plug J shown in Fig. 3 was identified as the most critical diagnostic port that requires special attention regarding radiation shielding.

Two-dimensional neutronics calculations have been performed to determine the nuclear radiation environment at selected locations in the diagnostics penetrations and to assess the impact of streaming on the average flux outside the port flange. Three different models were used in the calculations. The first one assumes no penetrations in the plug and flange. The results from this calculation are used as a reference to quantify the impact of streaming. The second case considered is for the worst case streaming with a $10 \mathrm{~cm}$ straight penetration through the plug and flange. This is representative of the NPA tube. The third case 
includes a penetration with four bends and represents the Thomson scattering laser well. The total neutron flux (integrated over all energies), the fast neutron flux $(\mathrm{E}>0.1 \mathrm{MeV})$ and the total gamma flux were calculated at the front of the plug, along the penetrations, at the back of the plug and at the back of the port flange. In addition, the absorbed dose rates in silica $\left(\mathrm{SiO}_{2}\right)$ and alumina $\left(\mathrm{Al}_{2} \mathrm{O}_{3}\right)$, were calculated. This includes the contributions from both neutrons and gammas. The gamma contribution to the dose varies from $\sim 30 \%$ at the front of the port plug to $\sim 80 \%$ at the back of the port flange.

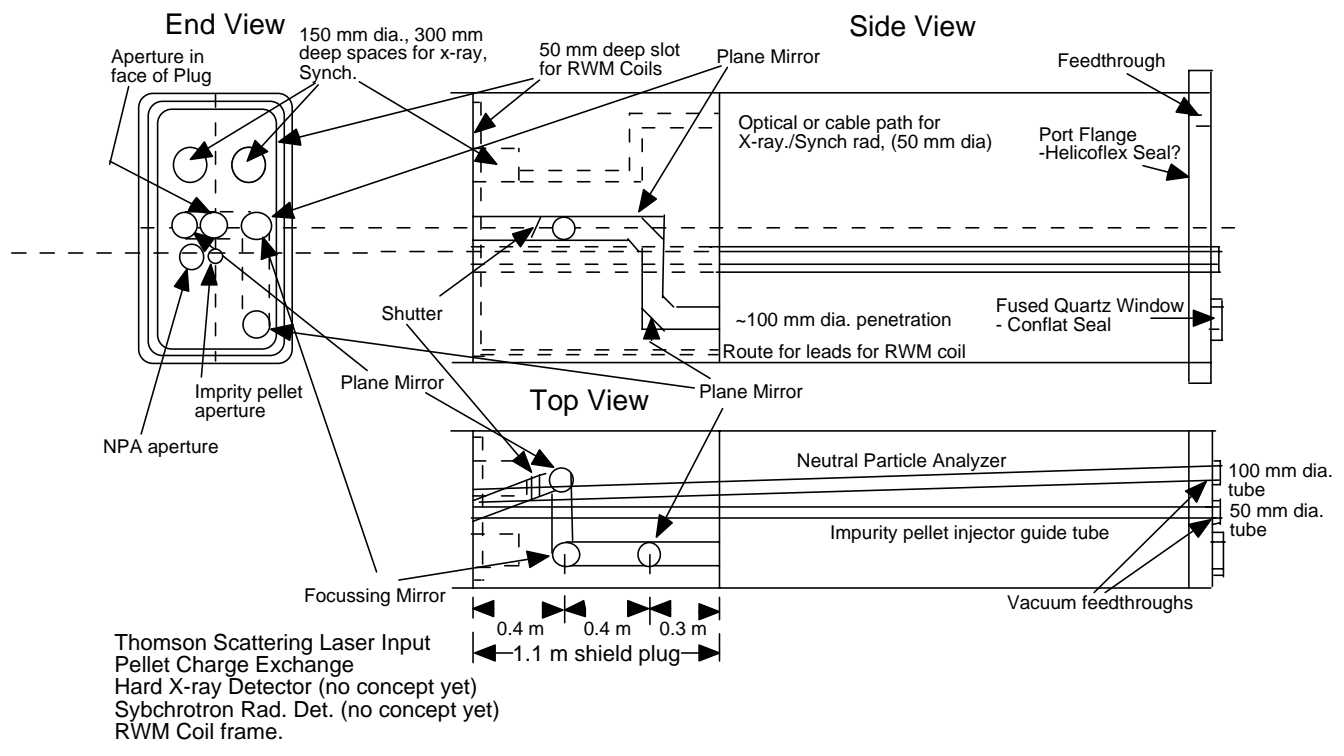

Fig. 3. Schematic of diagnostics and penetrations in port plug J.

Since the flux values in the test cell area behind the port flange are increased significantly due to streaming, the biological dose rate will be enhanced affecting the accessibility for hands-on clearing services prior to removal/installation of port assemblies by remote handling means. Acceptable biological dose rates behind the port flange were obtained from activation calculations performed with a $1.1 \mathrm{~m}$ port plug without any penetrations. These dose rates are $\sim 10 \mathrm{mrem} / \mathrm{hr}$ at shutdown, $\sim 3 \mathrm{mrem} / \mathrm{hr}$ after $1 \mathrm{hr}, \sim 0.1 \mathrm{mrem} / \mathrm{hr}$ after 1 day, and about $0.05 \mathrm{mrem} / \mathrm{hr}$ after 1 week. These acceptable dose rates can be maintained in the cases where penetrations are employed in the shield plug by increasing the plug thickness. Past activation calculations indicated that the dose rate scales linearly with the total neutron flux provided that the material used in the area where the dose is calculated is not changed. We performed two-dimensional neutronics calculations with different plug thicknesses for the cases with straight and 4-bend penetrations. For the straight penetration, the flux peaking factor increases from $\sim 4$ with $1.1 \mathrm{~m}$ plug to $\sim 15$ with $3.4 \mathrm{~m}$ plug. On the other hand, for the 4-bend penetration, the flux peaking factor increases from $\sim 2$ with $1.1 \mathrm{~m}$ plug to $\sim 13$ with $3.4 \mathrm{~m}$ plug. The average neutron flux behind the flange decreases by an order of magnitude by making the shield plug with straight penetration thicker by $\sim 0.5 \mathrm{~m}$. The same drop in flux is obtained by $\sim 0.42 \mathrm{~m}$ thicker plug for the case with 4-bend penetration. The results imply that if a straight $10 \mathrm{~cm}$ penetration is employed in the port plug, the plug thickness needs to be increased to $\sim 3.1 \mathrm{~m}$ to ensure that the dose rates after shutdown in the area behind the port flange are similar to the acceptable levels obtained without penetrations. For the case with the 4-bend penetration employed, the plug thickness should be increased to $\sim 2.1 \mathrm{~m}$. Since both penetrations exist in the diagnostic port $\mathrm{J}$, it is recommended that the port plug thickness should be increased to $3.4 \mathrm{~m}$, the location of the cryostat interface.

\section{Radwaste Assessment}

The radwaste of the different components of the machine were evaluated according to both the NRC 10CFR61 and Fetter waste disposal concentration limits. The waste disposal ratings (WDR) for the 10CFR61 and Fetter limits are shown in Table 4. These values are at the FIRE end-of-life with total of 5 TJ D-T and 
0.5 TJ D-D fusion energy. Results in the table are given for compacted wastes. Compacted waste corresponds to crushing the solid waste before disposal and thus disallowing artificial dilution of activity. The dominant nuclides are given between brackets. At the end of the machine life, all components would qualify for disposal as Class $\mathrm{C}$ low level waste according to the two waste disposal concentration limits used in the analysis. As shown in the table, according to Fetter limits, the WDR are dominated by the silver impurities in the CuCrZr alloy and the niobium impurities in the 316 SS and 304 SS alloys. The 10CFR61 limits indicate that the WDR of components made of the $\mathrm{CuCrZr}$ alloy are dominated by ${ }^{63} \mathrm{Ni}$ which is produced from copper by the ${ }^{63} \mathrm{Cu}(\mathrm{n}, \mathrm{p})$ reaction. On the other hand, the WDR of components made of the steel alloys are dominated by their niobium impurities. Due to the reduced neutron environment following D-D shots, all components will easily qualify for disposal as Class C LLW.

Table 4. Class C WDR.

\begin{tabular}{|l|c|c|}
\hline \multicolumn{1}{|c|}{ Zone } & Fetter & 10CFR61 \\
\hline IB FW & $0.17\left({ }^{108 \mathrm{~m}} \mathrm{Ag}\right)$ & $0.018\left({ }^{63} \mathrm{Ni}\right)$ \\
\hline IB VV & $0.076\left({ }^{108 \mathrm{~m}} \mathrm{Ag},{ }^{94} \mathrm{Nb}\right)$ & $0.029\left({ }^{94} \mathrm{Nb},{ }^{63} \mathrm{Ni}\right)$ \\
\hline IB Mag. & $1.63 \mathrm{e}-4\left({ }^{108 \mathrm{~m}} \mathrm{Ag}\right)$ & $9.13 \mathrm{e}-4\left({ }^{63} \mathrm{Ni}\right)$ \\
\hline OB FW & $0.17\left({ }^{108 \mathrm{~m}} \mathrm{Ag}\right)$ & $0.02\left({ }^{63} \mathrm{Ni}\right)$ \\
\hline OB VV & $8.8 \mathrm{e}-3\left({ }^{108 \mathrm{~m}} \mathrm{Ag},{ }^{94} \mathrm{Nb}\right)$ & $2.68 \mathrm{e}-3\left({ }^{94} \mathrm{Nb},{ }^{63} \mathrm{Ni}\right)$ \\
\hline OB Mag. & $1.88 \mathrm{e}-6\left(^{94} \mathrm{Nb}\right)$ & $2.12 \mathrm{e}-6\left({ }^{94} \mathrm{Nb},{ }^{63} \mathrm{Ni}\right)$ \\
\hline Divertor & $0.028\left({ }^{108 \mathrm{~m}} \mathrm{Ag}\right)$ & $0.011\left({ }^{94} \mathrm{Nb}\right)$ \\
\hline
\end{tabular}

The amount of radwaste generated in each of the chamber components is estimated in Table 5. It is worth noting that the WDR values are very low for the components that constitute the largest radwaste volume such as the magnets. These components might qualify for clearing or recycling leading to significant improvement in radwaste management.

Table 5. Amount of Chamber Radwaste.

\begin{tabular}{|c|c|c|}
\hline Zone & $\begin{array}{c}\text { Compacted Solid Waste } \\
\text { Volume }\left(\mathrm{m}^{3}\right)\end{array}$ & $\begin{array}{c}\text { Waste Mass } \\
\text { (tones) }\end{array}$ \\
\hline IB FW & $\begin{array}{l}0.08 \mathrm{Be} \\
0.6 \mathrm{Cu}\end{array}$ & $\begin{array}{l}0.15 \mathrm{Be} \\
5.4 \mathrm{Cu}\end{array}$ \\
\hline IB VV & $1.45 \mathrm{SS}$ & $11.5 \mathrm{SS}$ \\
\hline IB Mag. & $15.6 \mathrm{Cu}$ & $139.8 \mathrm{Cu}$ \\
\hline OB FW & $\begin{array}{l}0.14 \mathrm{Be} \\
1.5 \mathrm{Cu}\end{array}$ & $\begin{array}{l}0.26 \mathrm{Be} \\
13.4 \mathrm{Cu}\end{array}$ \\
\hline OB VV & $25.7 \mathrm{SS}$ & $203.5 \mathrm{SS}$ \\
\hline OB Mag. & $29.9 \mathrm{Cu}$ & $268.8 \mathrm{Cu}$ \\
\hline Midplane Port Plugs & $16.9 \mathrm{SS}$ & $133.8 \mathrm{SS}$ \\
\hline Divertor & $\begin{array}{c}0.14 \mathrm{~W} \\
0.8 \mathrm{Cu} \\
2 \mathrm{SS}\end{array}$ & $\begin{array}{c}2.7 \mathrm{~W} \\
7.2 \mathrm{Cu} \\
15.8 \mathrm{SS}\end{array}$ \\
\hline
\end{tabular}




\section{Recommended Future Work}

As FIRE proceeds beyond the current pre-conceptual design phase, more sophisticated neutronics analysis will be needed. Detailed three-dimensional modeling of the whole machine that includes material heterogeneity, penetrations and gaps is required to the level of detail used during the ITER engineering design activities. The model should also account for accurate plasma shape and source profile. The machine 3-D model will be the basis for performing detailed 3-D neutronics calculations to determine the different neutronics parameters (e.g., nuclear heating, radiation damage, insulator dose, etc.)

3-D modeling of ITER required a large amount of manpower because of the need to manually generate the parameters for the surfaces and elements in the geometrical input for the neutronics MCNP code. Significant savings in the modeling effort can be achieved if direct interface between the CAD drawings and the MCNP geometry module is developed. Development of codes that facilitate such an interface will be very valuable and will facilitate performing 3-D neutronics calculations with the ability to quickly accommodate design changes. Activities developing these interface codes were initiated at the University of Wisconsin during the pre-conceptual design phase of FIRE and need to expand as FIRE design proceeds to the conceptual design phase.

The 2-D analysis performed for the diagnostics port plug $\mathrm{J}$ showed that streaming is be an important issue even with the relatively small $10 \mathrm{~cm}$ penetration. 3-D analysis is essential to estimate the nuclear environment and radiation damage to the sensitive diagnostic system components (mirrors, fiber optics, critical electronics, etc.) in the diagnostic ports of FIRE. The impact of streaming through the diagnostics penetrations will have also to be evaluated due to its impact on accessibility for maintenance behind the cryostat.

The divertor region has a geometrically complex configuration that mandates 3-D calculations. Important issues that need to be addressed for that region are the amount of cryopump heating, nuclear heating and radiation damage in the divertor components, and neutron streaming through the divertor port.

The magnet insulator in FIRE is expected to experience significantly higher dose than that in ITER. That puts premier on the need for insulator R\&D program to qualify insulators for the high dose level expected in FIRE. In addition, we need to investigate the possibility of using better shielding material (e.g., W, WC, $\mathrm{B}_{4} \mathrm{C}$ ) to reduce the insulator dose particularly if extended FIRE operation is feasible.

In addition, more sophisticated activation analysis will be needed. The maintenance dose at critical locations with hands-on access (e.g., effect of penetrations in OB side on maintenance dose at cryostat) needs to be evaluated. Detailed multi-dimensional activation analysis will be required for more comprehensive safety assessment. 